\section{Acute longitudinal ligament rupture following acute spinal trauma}

\author{
Donald Hansom, Calan Mathieson, \\ Margaret Purcell, Duncan MacDonald \\ Department of Trauma Orthopaedic \\ Surgery, Royal Alexandra Hospital, \\ Paisley, UK
}

\begin{abstract}
The authors present a rare case of anterior longitudinal ligament (ALL) rupture in a 47year-old gentleman following a bicycle accident. The ALL is a continuous band of a variable thickness that acts as a primary spinal stabiliser. Stress, strain or rupture of the ALL usually occurs as a result of hyperextension, with the primary perpetrator being whiplash injuries. Such injuries have been shown to result in cervical spine instability during extension, axial rotation, and lateral bending modes. Spine radiographs of such patients may be routinely assessed as normal, therefore this specific type of injury does not lend itself to identification by traditional imaging methods. This account demonstrates the importance of having a high index of suspicion of a ligamentous neck injury in the setting of normal plain radiographs but abnormal clinical examination.
\end{abstract}

\section{Case Report}

A 47-year-old gentleman presented, following a bicycle accident whilst competing in a triathlon. The mechanism of injury involved crashing his bicycle at around 25-30 mph into a ditch after hitting a wet section of road on a tight bend. He landed directly onto the right frontal side of his face and outer aspect of his shoulder. At the scene there was no loss of consciousness, and his primary complaint was of right shoulder pain with pins and needles in his right index finger. He had no past medical history, was on no regular medications and had no allergies.

On initial presentation to Accident and Emergency Department, he was alert and orientated with a Glasgow Coma Score of 15/15. Routine observations were normal. Primary survey revealed superficial abrasions over his right shoulder and forehead with associated tenderness over his scapula. At this time he was noted to have a non-tender cervical spine, but was complaining of bilateral muscular pain around his neck. The rest of his primary survey was unremarkable, with no neurovascular deficit.

X-rays taken at this time revealed an un-displaced right scapular body fracture. Cervical spine films revealed no acute fracture and his cervical spine was initially cleared. It was not documented if he had head control or if neck movements produced pain.

Secondary survey carried out by the local orthopaedic team found the patient to have severe pain on attempted neck flexion. The patient himself felt that he had no head control. At this stage his neck was immobilised and full spinal protocol was followed. A computer tomography (CT) scan of his C-spine revealed no acute fracture, but did show a widening of the C4-5 disc space inferiorly with a slight posterior slip of C4 and C5 consistent with a subluxation at that level. This subluxation was also noted at the facet joints of C4-5 bilaterally (Figure 1). This raised the suspicion of an unstable ligamentous injury at this level. The CT scan also demonstrated bilateral first rib fractures and a medial end of clavicle fracture. Further magnetic resonance imaging (MRI) imaging confirmed disruption of the anterior longitudinal ligament (ALL) between C4-5 with increased signal between the anterior edge of the C4-5 disc and the upper endplate of C5. Associated posterior subluxation at this level was also noted (Figure 2). There was no radiological evidence of neurological injury. A well corticated bone fragment was also noted at the antero-superior aspect of $\mathrm{C} 6$ which was not felt to represent a new injury.

At this stage the patient was discussed with the local spinal injuries unit where he was subsequently transferred for further management. Flexion/extension views were performed which did not demonstrate significant instability and the patient was treated in a Miami-J collar. He was discharged from hospital 8 days after initial presentation. He remained in a collar for 3 months in total and at which stage further flexion extension views were obtained to confirm no late instability. On final assessment at four months post injury he could flex his chin to his chest without pain and had full neck extension. Lateral rotation was reduced to $45^{\circ}$ bilaterally. His shoulder function improved over this period and on final review he had an active/passive external rotation, abduction and forward flexion of $20^{\circ} / 30^{\circ}$, $90^{\circ} / 100^{\circ}$ and $100^{\circ}, 120^{\circ}$ respectively. He had full active and passive internal rotation.

\section{Discussion}

A relatively rare case of ALL rupture is presented. The spine has three main stabilising ligaments that act to provide a natural brace, protecting the spine from hyperextension/flex-
Correspondence: Donald Hansom, Department of Trauma Orthopaedic Surgery, Royal Alexandra Hospital, 0/14 Caird drive, Paisley G115DS, UK. Tel./Fax: +44.771.5419540.

E-mail: jdhansom@gmail.com

Key words: acute longitudinal ligament, acute spinal trauma, cervical spine.

Received for publication: 31 August 2013. Revision received: 29 January 2014.

Accepted for publication: 30 January 2014.

This work is licensed under a Creative Commons Attribution 3.0 License (by-nc 3.0).

(C) Copyright D. Hansom et al., 2014

Licensee PAGEPress, Italy

Emergency Care Journal 2014; 10:1903

doi:10.4081/ecj.2014.1903

ion injuries and aiding joint stability during rest and movement. ${ }^{1}$ These three ligaments are the ligamentum flavum, anterior longitudinal ligament (ALL) and posterior longitudinal ligament (PLL)

The ALL and PLL are continuous bands of a variable thickness that acts as primary spinal stabilisers, running from the basioccipital bone to the anterior aspect of the sacral promontory. ${ }^{2}$ Their key role is to prevent excessive movements of the vertebral bodies. The ALL functions to limit extension of the vertebral column and consists of a variety of different ligamentous fibres running from superficial to deep. The deepest fibres are short, intersegmental fibres, with the intermediate fibres spanning two or three vertebrae and the superficial fibres spanning three or four vertebrae. The ligament as a whole is therefore relatively thick and narrow over the vertebral bodies where it attaches to the periosteum. At the intervertebral disc, however, it widens and fibres bind directly to the fibrocartilage disc, the hyaline cartilage end plates and the margins of the vertebrae. ${ }^{2}$

The ALL plays a critical role in spinal stability. Stress, strain or rupture of soft tissue structures such as the ALL may be caused by relatively mild acceleration forces. ${ }^{3}$ Injury to the ALL usually occurs as a result of hyperextension, with the primary perpetrator being whiplash injuries. These injuries occur predominantly in rear-end vehicle crashes ${ }^{4,5}$ and impose a significant burden to both society and the individual. ${ }^{6}$ In these situations the occupant sustains postero-anterior acceleration to the torso, neck, and head structures secondary to rear-end impact from a vehicle or a fixed object. The neck-head structures respond with extension followed by flexion mechanism without the head impacting any interior object within the vehicle. This is an example of a noncontact acceleration injury 


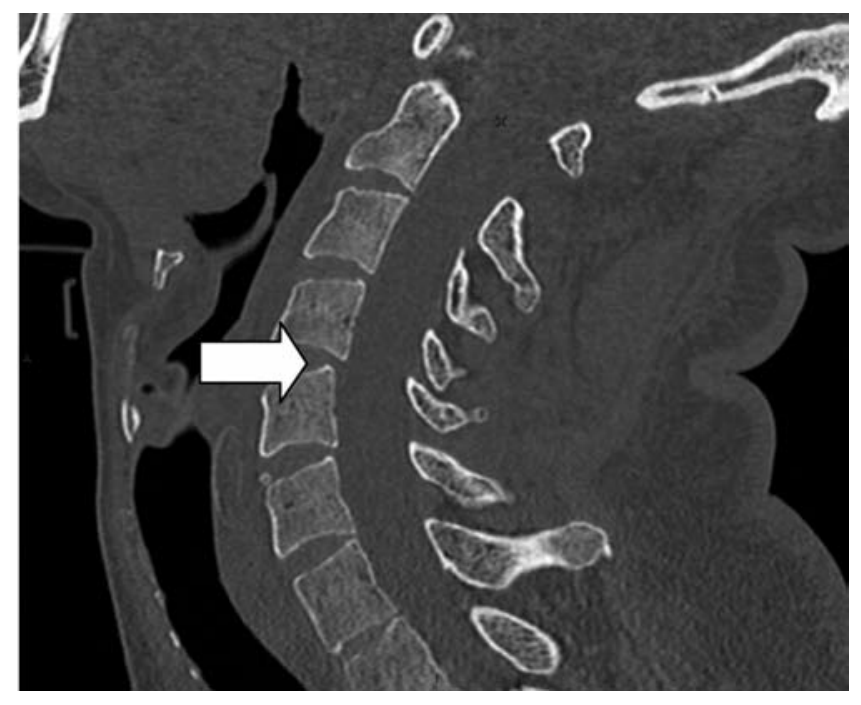

Figure 1. Non-contrast computer tomography scan. Arrow shows a widening of the anterior C4-5 disc space.

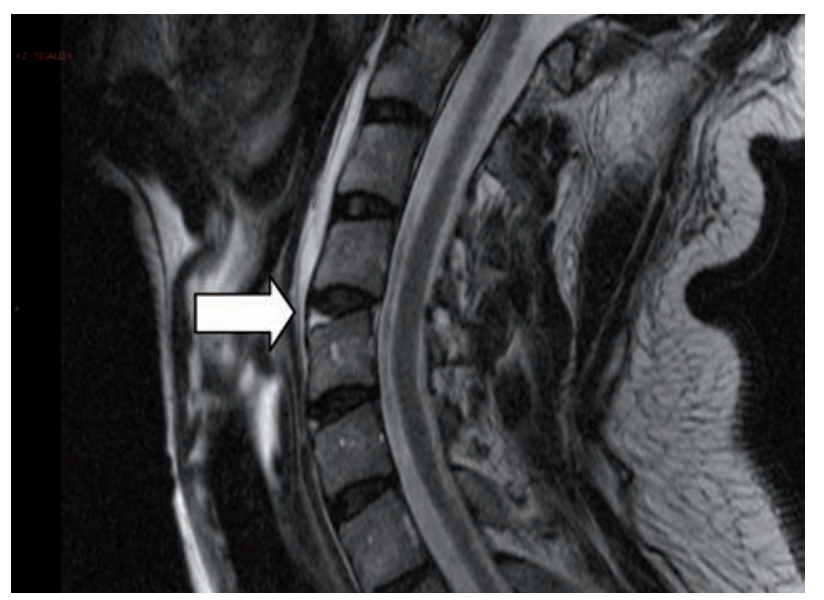

Figure 2. Magnetic resonance imaging C-spine. Arrow shows increased signal between the anterior edge of the C4-5 disc and the upper endplate of $\mathrm{C} 5$ with associated posterior subluxation. type known as inertial loading. ${ }^{6}$ During such hyperextension injuries the posterior bony elements of the vertebrae are compressed and occasionally fracture, while the anterior structures fail in tension resulting in a tear of the ALL or an avulsion fracture of the anterosuperior or anteroinferior edge of the vertebral body. The anterior part of the disc space opens up and sometimes a fracture of the back of the vertebral body and/or damage to the intervertebral disc is noted. ${ }^{3}$ Whilst whiplash injuries are common, and in some centres are present in up to $57 \%$ of all emergency accident cases, ${ }^{7}$ the incidence of associated ALL rupture is poorly documented. It is therefore postulated that despite there being a clear connection between ALL rupture and whiplash injury, the majority of whiplash patients do not suffer ALL rupture.

Such injuries have been shown to result in cervical instability during extension, axial rotation, and lateral bending modes ${ }^{8}$ however, the severity of impact is considered to be of low magnitude and often do not induce any bony damage in the form of acute fractures or subluxations. Spine radiographs of whiplashinjured patients are routinely assessed to be normal for the specific age group. Thus, this specific type of injury does not lend itself to objective identification by traditional imaging methods.

There are however several clinical decision rules that can be applied to this case which would have prevented our patients C-spine being cleared. The Canadian $\mathrm{C}$-spine rule (CCR) is designed to be used in stable trauma patients where a cervical spine injury is suspected and who have a GCS of $15 .{ }^{9}$ It evaluates suitable patients for one or more of following high risk criteria. If the patient is 65 years or older, they are deemed high risk. The second factor looks for any dangerous mechanism, including a fall from a height greater than 3 feet, a high-speed motor vehicle collision, an accident involving a bicycle/motorized recreational vehicle, or a direct axial load. The third high-risk factor is paresthesia in any or all extremities. If any three high-risk factors apply to the patient, the CCR mandates radiography. The National Emergency X-radiography Utilization Study (NEXUS) low-risk criteria was first introduced in $1992 .{ }^{10}$ In contrast, it highlights that cervical spine radiography may not be necessary to rule-out $\mathrm{C}$-spine injury in patients who displayed no cervical spine tenderness, signs of intoxication, altered mental status, or significant and painful distracting injuries. In retrospect, if these 2 clinical decision rules had been used, our patient would not have had his C-spine cleared following primary survey. His mechanism of injury would have been a high risk criteria in the CCR and he does not fulfil the NEXUS criteria as he has distracting injuries.

Our patient presented with several significant injuries including both a scapular and medial clavicle fracture on the right hand side and bilateral $1^{\text {st }}$ ribs fractures. Although initial $\mathrm{x}$-ray films showed no spinal fracture, the patient's other injuries suggested high energy trauma and when associated with a lack of head control, the suspicion of soft tissue injury was raised. Further CT and MRI imaging confirmed the diagnosis of ALL rupture. It is postulated that this rupture resulted from hyperextension trauma, causing a soft tissue fracture without bony injury. Although it is well documented in relation to road traffic accidents, ${ }^{4-6,11}$ little data is available for other injury mechanisms' such as that of our patient.

\section{Conclusions}

In conclusion, trauma patients presenting with multiple bony injuries suggestive of high energy with associated neck symptoms should be treated with a high degree of suspicion, regardless of $\mathrm{x}$-ray findings. The use of clinical decision rules such as CCR or NEXUS may be useful when deciding if a $\mathrm{C}$-spine can be cleared. We present an unusual case of soft tissue fracture by way of ALL rupture, and this should be a consideration in patients with symptoms of an unstable cervical spine without bony pathology in whom further imaging should be performed.

\section{References}

1. Colorado Spinal Institute, 2014. Understanding spinal anatomy. Available from: http://www.coloradospineinstitute. com/subject.php?pn=anatomy-ligaments17

2. Acland RD. Human anatomy. The trunk. Dvd 3. Philadelphia, PA, USA: Lippincott Williams \& Wilkins Publ.; 2003.

3. Stemper BD, Yoganandan N, Pintar FA, Rao RD. Anterior longitudinal ligament injuries in whiplash may lead to cervical instability. Med Eng Phys 2006;28:515-24.

4. Solomon L, Warwick D, Nayagam S. Apley's system review of orthopaedics and frac- 
tures. 9th ed. Abingdon UK: Hodder Arnold Publ.; 2010.

5. Spitzer W, Skovron M, Salmi L, et al. Scientific monograph of the Quebec task force on whiplash-associated disorders: redefining "whiplash" and its management. Spine 1995;20(Suppl.8):1S-73S.

6. Tennyson S, Mital NK, King AI. Electromyographic signals of the spinal musculature during $1 \mathrm{Gz}$ impact accelera- tion. Orthop Clin N Am 1977;8:97-119.

7. Yoganandan N, Cusick J, Pintar F, Rao R. Whiplash injury determination with conventional spine imaging and cryomicrotomy. Spine 2001;26:2443-8.

8. Ivancic P, Pearson A, Panjabi M, Ito S. Injury of the anterior longitudinal ligament during whiplash simulation. Eur Spine J 2004;13:61-8.

9. Galasko C, Murray P, Stephenson W.
Incidence of whiplash-associated disorder. B C Med J 2002;44:237-40.

10. Stiell IG, Wells GA, Vandemheen KL, et al. The Canadian C-spine rule for radiography in alert and stable trauma patients. JAMAJ Am Med Assoc 2001;286:1841-8.

11. Hoffman JR, Schriger DL, Mower W, et al. Low-risk criteria for cervical-spine radiography in blunt trauma: a prospective study. Ann Emerg Med 1992;21:1454-60. 